Bio - grafía. Escritos sobre la Biología y su Enseñanza. ISSN 2027-1034

Edición Extraordinaria. p.p. 1069 - 1077

Memorias del IX Encuentro Nacional de Experiencias en Enseñanza de la Biología y la

Educación Ambiental. IV Congreso Nacional de Investigación en Enseñanza de la Biología.

\title{
MODELOS EXPLICATIVOS DEL CONCEPTO DE SER VIVO EN ESTUDIANTES DE SEGUNDO DE PRIMARIA DE LA INSTITUCIÓN EDUCATIVA DISTRIT AL FRANCISCO DE MIRANDA DE BOGOTÁ, COLOMBIA.
}

\section{EXPLICATIVE MODELS OF LIVING BEING CONCEPT IN SECOND'S GRADE STUDENTS OF DISTRICT EDUCATIONAL INSTITUTION FRANCISCO DE MIRANDA OF BOGOTÁ, COLOMBIA}

\author{
MIRANDA M. \\ MÉNDEZ. A ${ }^{1}$
}

\section{RESUMEN}

Teniendo en cuenta, que los modelos explicativos son importantes para la enseñanza del concepto de "ser vivo" en las ciencias biológicas, por ser un punto de partida para pensar y planear la enseñanza, se realiza el presente estudio con el fin de Indagar y categorizar dichos modelos en los estudiantes de 6 a 8 años del grado segundo de primaria de la Institución Educativa Francisco de Miranda, Ubicada en la localidad de Kennedy de la ciudad de Bogotá. Este estudio se realizó bajo la implementación de la unidad didáctica Los seres vivos ¡Un mundo por descubrir! propuesta y diseñada por las investigadoras, teniendo en cuenta los postulados de Fonseca (2015), la cual permitió a través de su implementación, recolectar la información a través de herramientas propias de la investigación cualitativa como: diarios de campo e instrumentos de caracterización de ideas previas. El presente estudio se realizó enmarcado en el paradigma hermenéutico, por lo cual, los resultados fueron tratados bajo la propuesta de Bonilla (2013) y categorizadas en los modelos explicativos encontrados a través de la historia de la Biología: Animista, mecanicista, vitalista, organicista, neomecanicista y de pensamiento sistémico, encontrando que la mayoría de los estudiantes poseen modelos explicativos del concepto de ser vivo desde la perspectiva vitalista, animista y mecanicista.

PALABRAS CLAVE: Modelo explicativo, ser vivo, animismo, mecanicismo, vitalismo, neomecanicismo, sistémico.

\begin{abstract}
Taking into account that explanatory models are important for teaching the concept of "living being" in the biological sciences, as a starting point for thinking and planning teaching, the present study is conducted in order to investigate and categorize the models in the students of 6 to 8 years old of the second grade of the District Educational Institution

\footnotetext{
${ }^{1}$ Miranda. M. - Méndez. A. plzmiranda@gmail.com , mendez.alejandra96@gmail.com Universidad Distrital Francisco José de Caldas. Licenciatura en Biología.
} 
Bio - grafía. Escritos sobre la Biología y su Enseñanza. ISSN 2027-1034

Edición Extraordinaria. p.p. 1069 - 1077

Memorias del IX Encuentro Nacional de Experiencias en Enseñanza de la Biología y la

Educación Ambiental. IV Congreso Nacional de Investigación en Enseñanza de la

Biología.

Francisco de Miranda, located in the locality of Kennedy in Bogota city. This study was carried out under the implementation of the didactic unit The living beings $A$ world to discover! Proposed and designed by the researchers, taking into account the postulates of Fonseca (2015), which allowed through the implementation, the information was collected through tools of research qualitative as: field journals and instruments of characterization of previous ideas. The present study was framed in the hermeneutic paradigm, so the results were treated under the proposal of Bonilla (1995) and categorized in explanatory models found throughout the history of Biology: Animist, mechanistic, vitalist, organic, Neo-mechanicist and systemic thinking, finding that most of the students have explanatory models of the concept of living being from the vitalist, animistic and mechanistic perspective.

KEY WORDS: Explanatory model, living being, animism, mechanicism, vitalism, neomechanicism, systemic.

\section{INTRODUCCIÓN}

Según Tamayo, Orrego \& Davila (2014), los modelos explicativos desde la didáctica de las ciencias, están orientados a conocer cuáles son las representaciones internas que tienen los educandos en dominios específicos del conocimiento, tanto los que hacen referencia al orden intuitivo como los adquiridos mediante la enseñanza. El estudio de los modelos explicativos, se basa en comprender y reconocer la existencia de estos en los estudiantes, conociendo su forma de representación mental y cómo son empleadas por los profesores en el aprendizaje significativo, el cual le permite al estudiante relacionar la información nueva con la que ya posee; reajustando y reconstruyendo ambas informaciones en este proceso (Ausubel, 1973). Según Gilbert \& Boulter (2000), el uso de los modelos explicativos de los estudiantes permite crear estrategias de enseñanza de las ciencias e identificar los obstáculos frente al aprendizaje, el cual pretende ser significativo.

Debido a su intencionalidad descriptiva, los modelos explicativos han sido empleados como estrategias de enseñanza y de aprendizaje. Por lo cual, en el campo de la didáctica de las ciencias se han realizado diversos trabajos concernientes a los modelos explicativos en estudiantes, como: El movimiento de la tierra (Vosniadou \& Brewer, 1992), magnetismo (Greca y Moreira, 1998), cambio químico (Khan, 2008), entre otros; en el campo de la biología se encuentran aportes en bioenergética (Tamayo, 2001, Tamayo \& Sanmartì, 2007), inmunología (Orrego, López, y Tamayo, 2012), respiración (Nuñez y Banet 1996), fotosíntesis (Waheed \& Lucas, 1992), entre otros.

De acuerdo con Barney, Torres y Villa (2009), el conocimiento amplio de la historia de la ciencia, permite reconocer los modelos explicativos que se han venido gestando a lo largo de la misma. Por su parte, Azcaraté (1984), propone un tratamiento didáctico por medio de la historia del concepto, en estudios sobre la comprensión de las dificultades en la 
Bio - grafía. Escritos sobre la Biología y su Enseñanza. ISSN 2027-1034

Edición Extraordinaria. p.p. 1069 - 1077

Memorias del IX Encuentro Nacional de Experiencias en Enseñanza de la Biología y la

Educación Ambiental. IV Congreso Nacional de Investigación en Enseñanza de la Biología.

elaboración del modelo explicativo de velocidad y aceleración, por parte del científico Galileo, relacionándolo con los problemas de aprendizaje que presentan sus estudiantes con estos conceptos; de la misma manera autores como Solbes y Travel (2001), dan cuenta de la importancia de la historia de las ciencias como herramienta didáctica para el aprendizaje del concepto, generando en los educandos mayor comprensión y motivación. Sin embargo Valera (1983), es quien relaciona las ideas de sus estudiantes, con los modelos explicativos correspondientes a periodos históricos de la ciencia, partiendo de la tesis de que dichas ideas, son construidas a partir de la observación de los fenómenos naturales. Lo anterior, deja en evidencia que es importante tener en cuenta la historia del concepto de ser vivo, tanto para enseñarlo como para comprender los modelos explicativos que se han gestado a lo largo del tiempo. Por esta razón, se eligieron para el presente estudio, los modelos explicativos del concepto de ser vivo a partir de la historia de las ciencias, con el fin de clasificar en ellos, lo dicho por los estudiantes de segundo de primaria acerca del concepto.

Teniendo como base el desarrollo histórico del concepto de ser vivo, se han encontrado diversos modelos para su estudio, los cuales son: el animismo, el mecanicismo, el vitalismo, el organicismo, el pensamiento sistémico y el neomecanicismo. Según Correa (2009), el modelo animista surgió en la edad antigua con el filósofo griego Aristóteles. Se caracteriza porque en este modelo se considera a los seres vivos como aquellos que poseen movimiento, centrando sus creencias en la divinidad, estas creencias fueron populares en las sociedades primitivas. El modelo mecanicista, inició en el siglo XVIII con la física mecánica y las leyes newtonianas del movimiento. En este modelo se da importancia al funcionamiento de los cuerpos y todo el movimiento del que se encuentran provistos. El modelo vitalista, se reconoce con Leibniz, relacionando el concepto de materia con el de fuerza activa o fuerza vital. El desarrollo de un organismo se da por medio de procesos que llevan una secuencia y una autorregulación. Este modelo se basa en el desarrollo que tiene un ser vivo, teniendo en cuenta todo su proceso de organización. El modelo organicista, inicia en el siglo XX con Ross Harrison, en este modelo se dan a conocer los procesos vitales a nivel molecular. El estudio de la organización de los seres vivos desde su composición, permite hacer explicaciones por mecanismos físico-químicos, sin embargo, este modelo no alcanzó su integración, debido a sus niveles de organización. El modelo pensamiento sistémico, comparte los postulados del organicismo, planteando como sistemas vivos a los organismos en sus relaciones, siendo comprendidos desde la organización del conjunto, por lo cual no se puede dividir las partes y realizar un análisis de ellas. Por último, el modelo neomecanicista, explica lo vivo a partir de la estructura que los compone y su parte macromolecular. De ello nacen varias ramas de la biología que estudia la estructura de los seres vivos, como la genética.

En cuanto a la enseñanza del concepto de vida. Piaget (1933), fue uno de los primeros académicos en realizar estudios del concepto de vida en niños, haciendo énfasis en el desarrollo del concepto a partir de la relación del sujeto con otros seres vivos y su edad psíquica. En el año 1976, Piaget concluye en otro de sus estudios, que la transformación del concepto en el sujeto, se da de forma secuencial a medida que va descubriendo y ampliando la mirada de su mundo, caracterizando estas observaciones en cuatro estadios 
Bio - grafía. Escritos sobre la Biología y su Enseñanza. ISSN 2027-1034

Edición Extraordinaria. p.p. 1069 - 1077

Memorias del IX Encuentro Nacional de Experiencias en Enseñanza de la Biología y la

Educación Ambiental. IV Congreso Nacional de Investigación en Enseñanza de la

Biología.

principales clasificados bajo el criterio de la edad física: de los 4 a los 6 años, se considera vivo, cualquier objeto o ser que tenga alguna función o realice alguna actividad. De los 6 a 8 años, la locomoción es fundamental en el desarrollo del concepto, sin importar la forma en la cual se da la movilidad. De los 8 a los 10 años, se configura un nuevo aspecto relativo a la espontaneidad y autonomía en los cuerpos móviles y desde los 11 años, la vida se categoriza principalmente como una característica exclusiva de plantas y animales.

Basados en Piaget, otros autores realizan estudios acerca de la percepción de vida, presentando algunos resultados novedosos atendiendo a la facultad de movimiento, como Benzonsky (1974) y Lucas, Linke y Sedgwik (1979). Kelemen (1999) en cambio en su conclusión referente al raciocinio humano, en el cual, explica que la capacidad de razonar en términos físicos y causales, es nula en los primeros años del desarrollo.

Para Castaño. N.C. y Leudo, M. (1998), entre los 5 y 7 años, los niños transforman su pensamiento animista, reconociendo en el movimiento autónomo una característica esencial de lo vivo, con lo cual se empieza a diferenciar el tipo de movimiento de un cuerpo entre autónomo y condicionado, reconociendo así, que la facultad del ser vivo se encuentra en los movimientos propios que no requieren de una batería. De forma específica, de los 5 a los 6 años, los estudiantes empiezan a utilizar términos con alguna connotación biológica como lo es crecer o reproducirse, también se utilizan rasgos autopomórficos para afirmar o negar el carácter de ser vivo, así como concepciones utilitaristas, creacionistas, animistas, de identificación de artefactos, racionalistas y nociones del requerimiento de energía y cuidado. A los 7 años, se empiezan a considerar a los animales como seres vivos, sin embargo, aún las plantas están desprovistas de tal facultad, al ser consideradas como "palos" y a los 8 años, tanto plantas como animales, empiezan a ser considerados como seres vivos, al asignarles el carácter de activo y la designación de objetos inanimados en el movimiento. Evidenciando en esta etapa como el movimiento deja de ser esencial para hacer las consideraciones de ser o no vivo.

En el estudio de Corrêa, Meglhioratti y De Andrade (2010), se analizan las ideas de vida surgidas en clase para discutirlas, integrarlas y articularlas con las definiciones de vida teóricamente fundamentadas como lo son: La vida como autopoiesis (Maturana y Varela, 1973), explicada desde las interconexiones moleculares organizadas y capaces de mantener sus sostenibilidad de manera autónoma, la vida como selección de explicaciones (Emmeche y El-Hani, 2000), en la cual el ambiente actúa relacionando entidades dentro de una serie de replicaciones, que cumplen el mismo patrón de la selección natural, en la selección de genes y selección de organismos y la vida como interpretación de signos (Ruiz-Mirazo et al. 2004), que busca en la interpretación de los signos de la naturaleza una explicación que pretende entender el concepto de vida por medio de una semiótica.

\section{METODOLOGÍA}

El estudio se realizó bajo el ámbito de la investigación cualitativa, en la cual, el 
Bio - grafía. Escritos sobre la Biología y su Enseñanza. ISSN 2027-1034

Edición Extraordinaria. p.p. 1069 - 1077

Memorias del IX Encuentro Nacional de Experiencias en Enseñanza de la Biología y la

Educación Ambiental. IV Congreso Nacional de Investigación en Enseñanza de la Biología.

investigador debe involucrarse con la población de estudio para tener una mejor interpretación de los acontecimientos, e identificando puntos críticos presentados en distintas áreas que afectan a las poblaciones, así pues, la interpretación de la realidad se da de forma descriptiva, analítica, crítica, y objetiva (Bonilla, 1995).

La investigación se llevó a cabo en la Institución Educativa Distrital (I.E.D.) Francisco de Miranda, en el barrio Timiza ubicado en la localidad de Kennedy de la ciudad de Bogotá, en el grado segundo, el cual está conformado por 36 estudiantes que se encuentran entre las edades de 6 a 8 años.

En el marco de la implementación de la unidad didáctica: Los seres vivos ¡Un mundo por descubrir!, la cual presenta una propuesta para la enseñanza del concepto "Ser vivo" por parte de los investigadores, de acuerdo a Fonseca (2015) y el modelo aprendizaje significativo (Ausubel, 1978); se realizaron 6 clases en las cuales se recolectó información correspondiente a los modelos explicativos de los estudiantes concernientes al concepto "Ser vivo".

Los resultados obtenidos en las fichas de caracterización de ideas previas y en el diario de campo, fueron tratados bajo los parámetros propuestos por Bonilla (1995), los cuales permitieron un proceso de interpretación de los mismos, para ubicarlos en las categorías de los modelos explicativos encontrados del estudio histórico del concepto "ser vivo", como lo son: el Vitalista, Mecanicista, animista, organicista, Neomecanicista y de pensamiento sistémico, desarrollados por Correa (2009).

Los resultados fueron tratados bajo las condiciones de Bonilla (1995), en la cual propone que los datos cualitativos deben ser categorizados y descritos, la revisión de los datos deben estar basados en la búsqueda de evidencias que corroboren o invaliden los supuestos que guían el trabajo, la formulación de explicaciones sobre el fenómeno y la identificación de los esquemas teóricos.

\section{RESULTADOS Y ANÁLISIS}

De acuerdo a los resultados obtenidos los modelos explicativos encontrados en los estudiantes para explicar el concepto de ser vivo son: Vitalismo, Animismo y Mecanicismo. A continuación, se hace referencia a cada uno de los modelos mencionados.

Algunos de los aspectos característicos del modelo animista son: la asignación de alma la cual provee de todas las cualidades de la vida, y esta es dada por una divinidad. Se encontró que el $12 \%$ de la población estudiantil se ubica en este modelo (grafico 1). Algunas de las expresiones que se muestran a continuación ilustran lo antes planteado.

Estudiante 1: "Dios creo todo lo vivo y lo no vivo".

Estudiante 2: "Dios nos creó a su imagen".

Estudiante 3: "Dios decide que está vivo y muerto". 
Bio - grafía. Escritos sobre la Biología y su Enseñanza. ISSN 2027-1034

Edición Extraordinaria. p.p. 1069 - 1077

Memorias del IX Encuentro Nacional de Experiencias en Enseñanza de la Biología y la

Educación Ambiental. IV Congreso Nacional de Investigación en Enseñanza de la Biología.

Estudiante 4: "Dios es nuestro padre por eso tengo vida".

Entre los aspectos que caracterizan el modelo animismo se encuentra el creacionismo, que, de acuerdo con el desarrollo histórico, en el siglo XVI, se consideraba que las especies eran el resultado de la creación divina de un Dios. Entre algunas respuestas se evidencia que los estudiantes consideran a los seres vivos, como creación a imagen y semejanza de un Dios (divinidad), el cual dota de vida además de todas las características y cualidades para su subsistencia.

El modelo mecanicista se caracteriza de acuerdo a la analogía cuerpo-máquina que según Barcarlett y Fuentes (2007): la maquina es una construcción artificial, obra del hombre, en la cual la función esencial depende de mecanismos, el automatismo designa la construcción de aparatos imitadores de movimientos orgánicos y de acción, aparte que su construcción y disparo inicial es hecho por el hombre", esta analogía hace referencia al movimiento que realiza un cuerpo y una máquina. En este modelo se consideran aquellas expresiones de los estudiantes en las cuales se afirma que los seres vivos son todos aquellos que están provistos de movimiento el cual les permite vivir $(56 \%$ de las respuestas) (Gráfico 1). En otras palabras, el movimiento se considera algo natural y propio por tanto debe realizarse.

Estudiante 5: "Los seres vivos se mueven"

Estudiante 6: "Están vivos porque caminan y corren o saltan como yo"

Estudiante 7: "Para vivir se necesita mover"

Estudiante 7: "Es algo con lo que nacemos y es necesario moverse"

Al preguntarles entonces si las plantas no eran seres vivos debido a que no presentaban movimiento algunos de ellos respondían que las plantas si están vivas por el hecho de crecer y alimentarse de la tierra. Por tanto, como se ve evidenciado hay un choque en las explicaciones ya que se muestra confusión al explicar por qué las plantas son seres vivos si estas no poseen movimiento.

Estudiante 8: "Las plantas si viven porque crecen mucho y necesitan agua"

Estudiante 9: "Están vivos porque los árboles duran mucho"

Estudiante 10: "Las plantas necesitan agua porque si no se mueren"

Estudiante 11: "Nosotros y las plantas necesitan agua por eso estamos vivos"

Al reconocer que el movimiento es un proceso que todo organismo necesita realizar se pasan por alto aquellos organismos que no poseen esta capacidad. En este caso se observa una explicación desde aspectos macroscópicos. En este modelo ubican en un primer plano la naturaleza de los seres vivos y justifican el proceso del movimiento desde allí.

El modelo vitalista se centra en explicar el desarrollo de un organismo, este desarrollo se da por medio de procesos, que llevan una secuencia, una autorregulación, de allí, el 
Bio - grafía. Escritos sobre la Biología y su Enseñanza. ISSN 2027-1034

Edición Extraordinaria. p.p. 1069 - 1077

Memorias del IX Encuentro Nacional de Experiencias en Enseñanza de la Biología y la

Educación Ambiental. IV Congreso Nacional de Investigación en Enseñanza de la Biología.

vitalismo se basa en el desarrollo que tiene un ser vivo (Correa, 2009). En este modelo se evidencia que los estudiantes explican que los seres vivos tienen un proceso en el cual nacen, crecen, se reproducen y mueren, las respuestas que los estudiantes presentan están muy ligadas también al modelo mecanicista, basándose en el desarrollo que tiene un ser vivo para poder subsistir. A los seres vivos (biocenosis) se les identifica como aquellos que "se mueven, comen y crecen" y a los seres no vivos (biotopo) se les identifica como aquellos que "no se mueven, no hablan y tampoco crecen". El $32 \%$ de las respuestas de los estudiantes se ubicaron en este modelo explicativo (Gráfico 1).

Estudiante 12: "Todo lo que está vivo nace, crece y se mueren"

Estudiante 13: "Se debe comer para crecer y vivir"

Estudiante 14: "Lo que no está vivo no crece, como las piedras"

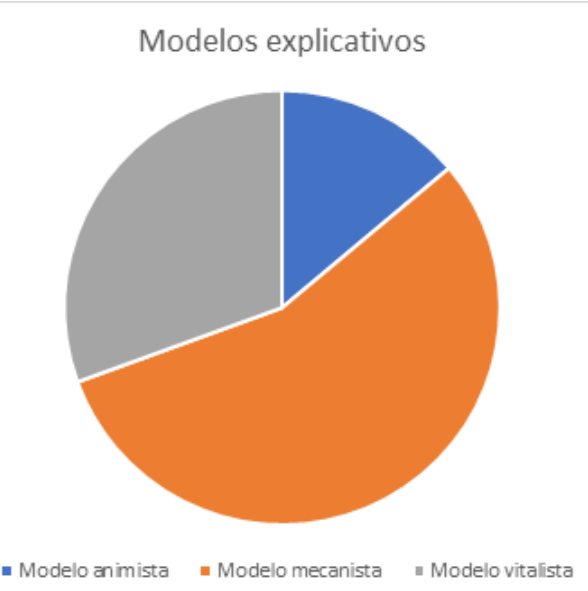

Gráfico 1. En este gráfico se presentan los modelos explicativos que presentó la población total de estudiantes la cual consta de 36. En el modelo animista se encuentran el $13 \%$ de la población (11 estudiantes). En el modelo mecanicista está el $56 \%$ de la población $(20$ estudiantes) $y$ en el modelo vitalista se encuentra el $31 \%$ de estudiantes $(11$

estudiantes).

\section{CONCLUSIONES}

Para la enseñanza de las ciencias la descripción de los modelos explicativos de los estudiantes es un punto de partida para pensar y planear la enseñanza. Para la caracterización de los modelos se necesita un acercamiento desde la disciplina específica y desde el ámbito de enseñanza y aprendizaje. En este sentido los modelos permiten observar los obstáculos para la enseñanza de las ciencias y su valor reside fundamentalmente en cómo usarlos, constituyéndose como el punto de partida de los procesos de enseñanza.

Se encontró en los estudiantes los modelos: vitalista, animista y mecanicista como principales modelos explicativos lo cual corresponde con la idea piagetiana en el 
Bio - grafía. Escritos sobre la Biología y su Enseñanza. ISSN 2027-1034

Edición Extraordinaria. p.p. 1069 - 1077

Memorias del IX Encuentro Nacional de Experiencias en Enseñanza de la Biología y la

Educación Ambiental. IV Congreso Nacional de Investigación en Enseñanza de la Biología.

mecanicismo y los postulados de Castaño et al (1998), en el vitalismo y animismo, del concepto de "ser vivo" en estudiantes de 6 a 8 años.

\section{REFERENCIAS}

- AUSUBEL, D. P. (1973). "Algunos aspectos psicológicos de la estructura del conocimiento". En Elam, S. (Comp.) La educación y la estructura del conocimiento. Investigaciones sobre el proceso de aprendizaje y la naturaleza de las disciplinas que integran el currículum. Ed. El Ateneo. Buenos Aires. Págs. 211-239.

- BONILLA, E. Rodríguez, V. (1995). La investigación en ciencias sociales: Más allá del dilema de los métodos. Primera edición, Centro de Estudios para el Desarrollo Económico (CEDE). Facultad de Economía, Universidad de los Andes. Bogotá, Colombia.

- CASTAÑO, N. C. y Leudo, M. (1998). Nociones de los niños acerca de lo vivo: Implicaciones didácticas. Universidad Pedagógica Nacional. Bogotá, Colombia.

- CORREA, A. Meglhoratti A. De Andrade, A. (2010). CONCEITO DE VIDA: UMA PROPOSTA PARA O ENSINO DE CIÊNC IA NA EDUCAÇÃO FUNDAMENTAL. VII Encuentro Nacional de pesquisa em educaÇÃo em Ciéncias. Rev. Florianópolis. ISSN 21766940. Brasil.

- CORREA, M. (2009). El interrogante de lo vivo y sus respuestas ahistóricas: desarrollo histórico del concepto de lo vivo y algunas implicaciones didácticas. Biografía: escritos sobre la biología y su Enseñanza. Vol. 2 N 1 ISS 2027 - 1034.

- EL-HANI, C. N. Theory-based approaches to the concept of life and biological education. Journal of Biological Education, 42(4): 147-149.

- EMMECHE, C. EL-HANI, C. (2001). Definindo vida: O que é vida? Para entender a Biologia do século XXI, Editora Relume Dumará, 2 ed. Rio de Janeiro, Brasil.

- FONSECA, G. (2015). GUIA DE TRABAJO: DISEÑO DE UNIDAD DIDÁCTICA. Universidad Distrital Francisco José de Caldas.Bogotá, Colombia.

- MATURANA, H.; Varela, F.J. (1980). Autopoiesis and cognition: the realization of the living, BSPS, vol. 42, D. Reidel, Boston, Estados Unidos.

- MATURANA, H.; Varela, F.J. (2009). De Máquinas Y Seres Vivos: Autopoiesis, La Organización De Lo Vivo. Editorial Universitaria LUMEN, 6ta ed. Buenos Aires, Argentina.

- PIAGET, J. (1933). La representación del mundo en el niño. Morata 7ma edición. Madrid.

- PIAGET, J. (1976) La toma de conciencia. Editorial Madrid: Morata. España. 
Bio - grafía. Escritos sobre la Biología y su Enseñanza. ISSN 2027-1034

Edición Extraordinaria. p.p. 1069 - 1077

Memorias del IX Encuentro Nacional de Experiencias en Enseñanza de la Biología y la Educación Ambiental. IV Congreso Nacional de Investigación en Enseñanza de la Biología.

- tamaYo, O. Orrego, M. Dávila, A. (2014). MODELOS EXPLICATIVOS DE ESTUDIANTES ACERCA DEL CONCEPTO DE RESPIRACIÓN. Bio-grafía Escritos sobre la Biología y su enseñanza Vol. 7 - No.13 pp. 129 - 145.

- TAMAYO A. O. (2001). Evolución conceptual desde una perspectiva multidimensional. Aplicación al concepto de respiración. Tesis doctoral. Universidad Autónoma de Barcelona. Barcelona. 